\title{
Educação e Humanidades em saúde: a experiência do grupo de Humanidades do curso de Medicina da Universidade Estadual do Ceará (Uece)
}

PALAVRAS-CHAVE:

-Humanidades;

-Educação Médica;

-Relações Médico-Paciente.

KEYWORDS

-Humanities;

-Medical Education;

-Physician Patient Relationship

Recebido em: 27/07/2007

Reencaminhado em: 13/09/2007

Aprovado em: 22/09/2007

\author{
Education and Humanities in health: the \\ experience of the Humanities group of the \\ medical course, Federal University of the \\ State of Ceará (Uece)
}

Kathiane Lustosa Augusto ${ }^{1}$ Carolina Arcanjo Lino Amanda Gisele Nobre Carvalho ${ }^{1}$ Carlos Maximiliano Gaspar Carvalho Heil Silva ${ }^{1}$ Filipe Castro de Andrade Natália Braga Hortêncio Jucá ${ }^{1}$ Andréa Caprara $^{1}$

\section{RESUMO}

Nos últimos anos, vem se desenvolvendo uma grande área de reflexão e pensamento, denominada "humanidades médicas", que incorpora a realidade social e a experiência individual à interface entre médico e paciente. O grupo Humanidades, Saberes e Práticas em Saúde nasce em 2004, como núcleo de desenvolvimento de pesquisas, composto por estudantes de Medicina, com o objetivo de explorar como a prática médica lida com as experiências de pacientes e de médicos e o processo saúde-doença. As linhas de ação envolvem pesquisa com médicos oncologistas e sua visão da relação médico-paciente na consulta oncológica e ensino no terceiro e quarto semestres do curso de Medicina, utilizando casos clínicos, modelo teórico e role play. Nessa perspectiva, busca-se aproximar a temática da relação médico-paciente do cotidiano dos estudantes de Medicina, contribuindo para desenvolver uma atitude humanizada frente ao ser humano portador de enfermidade.

\begin{abstract}
During the past few years a great area of thought and reflection named medical humanities, incorporating social reality and individual experience to the doctor-patient relationship, has evolved. The group Humanidades, Saberes e Práticas - Humanities, Knowledge and Practices - was born in 2004 as a research nucleus composed by medical students, with the intent of getting insight into how the medical practice is dealing with the experience of doctors and patients and with the health-disease process. The lines of action involve research with oncologists for obtaining their view about the doctor-patient relationship in the oncological consultation as well as about the use of clinical cases, theoretic model and role play as teaching strategies for medical students from the third and fourth semesters. From that perspective we are trying to approximate the issue doctor-patient relationship and the every-day routine of medical students in an attempt to contribute to the development of a humanized attitude towards the diseased human being.
\end{abstract}




\section{INTRODUÇÃO}

A prática médica vem passando por profundas mudanças desde as décadas de 1970 e 1980, entre elas um extraordinário aumento da produção do conhecimento científico, a necessidade de novas capacidades profissionais, de raciocínio clínico, de comunicação e de negociação com os pacientes, o uso de novas tecnologias de informação, um aumento da capacidade de compreensão dos problemas sociais e culturais das comunidades e novos questionamentos éticos ${ }^{1}$.

Hoje se sabe que muitos conhecimentos não são utilizados na prática e que é impossível incluir em um programa de Medicina todas as informações necessárias. Os conhecimentos e as práticas médicas mudam rapidamente, e isso implica um processo de aprendizagem que ajude estudantes e docentes a estarem constantemente atualizados. Estas mudanças acarretam uma transformação da Educação Médica para que consiga aumentar a capacidade de auto-aprendizagem dos alunos das Faculdades de Medicina, melhorar as capacidades clínicas e de tomada de decisões, melhorar o relacionamento com os pacientes e acrescentar novas visões sociais e culturais da relação saúde-doença, numa ótica de prevenção e promoção à saúde.

A identificação dessas necessidades não é nova, e vários autores no passado apresentaram análises detalhadas e completas a este respeito ${ }^{1,2,3}$. No Brasil, formou-se em 1991 uma Comissão Interinstitucional Nacional de Avaliação do Ensino Médico, a Cinaem, que apresentou em 1997 seu relatório geral. Os resultados desse trabalho constituem um ponto importante de referência, mostrando como o modelo pedagógico atual é inadequado em vários aspectos. Entre estes, os autores enfatizam a forma irracional de incorporação da tecnologia, maior ênfase no ensino e no repasse da informação do que no processo de aprendizagem, falta de uma formação ético-humanista, ênfase na especialização médica e nas ações curativas em prejuízo daquelas relacionadas à promoção e prevenção das doenças ${ }^{4}$.

Segundo os resultados dessa Comissão, o processo de formação deve se concentrar menos no ensino e mais no processo de aprendizagem; a grande quantidade de informações, sempre mais disponíveis, deve priorizar o processo de ensino-aprendizagem, em função de sua aplicabilidade na prática clínica e na promoção e prevenção das doenças; durante todo o processo de formação, as ciências básicas devem estar integradas à parte clínica; a parte biológica deve ser acompanhada pela compreensão dos aspectos sociais e culturais do processo saúde-doença, incluindo, além dos hospitais, os consultórios comunitários; o processo de avaliação deve ser coerente com os objetivos educacionais ${ }^{4,5}$.
Nos últimos anos, vem se desenvolvendo uma grande área de reflexão e pensamento denominada "humanidades médicas". As novas matrizes curriculares teriam que incorporar elementos das ciências humanas (filosofia, psicologia, antropologia, literatura) aos cursos de graduação e especialização. Essa incorporação permitiria ampliar a sensibilidade e a compreensão do paciente ${ }^{6}$. A inserção das Humanidades em currículos de profissionais de saúde responde à necessidade de humanizar o produto final desses currículos ${ }^{7}$.

As humanidades médicas permitem desenvolver uma nova compreensão da vivência e do sofrimento da pessoa, incorporando a realidade social e a experiência individual à interface entre médico e paciente. Trata-se de uma concepção integrada que pretende melhorar as capacidades de comunicação dos médicos e aprofundar a narrativa sobre enfermidade, procurando novas formas de promoção do bem-estar do paciente ${ }^{8}$.

Para isso, porém, a contradição entre formação de especialistas e generalistas começa a exigir que a Educação Médica enfrente criticamente a determinação tecnológica do critério médico de qualidade, que envolve tanto a ética profissional como a eqüidade. É necessário dar continuidade à formação de bons especialistas e, ao mesmo tempo, resgatar e fortalecer a formação geral na graduação, inserindo-a adequadamente nas equipes de saúde, promovendo também suas funções e reconhecimento sociais ${ }^{9}$. A formação de profissionais no modelo biopsicossocial tem se mostrado mais adequada para a obtenção de práticas humanizadas, que permitam compreender o universo psicológico do paciente ${ }^{10}$.

Aguiar propõe que os conhecimentos científicos básicos de natureza biopsicossocial subjacentes à prática médica são necessários ao exercício profissional não autoritário, capaz de negociar condutas e intervenções a partir da escuta atenta das perspectivas de pacientes, famílias e comunidade ${ }^{11}$. A Educação Médica passa, então, a ter de se preocupar em formar tanto médicos preparados nos aspectos técnicos da doença, como também cuidadores humanizados, sensíveis para lidar consigo e com seus pacientes, tarefa que exige trabalhar com os mais diversos valores inseridos em complexos contextos históricos, culturais e sociais.

No entanto, durante a formação, os alunos aprendem que os sentimentos são algo de que se devem afastar. Eles devem manter a "neutralidade", precisam vestir um manto imaginário que os proteja das emoções. É preciso modificar essa imagem de "médico ideal", segundo a qual as emoções estão excluídas, para facilitar aos futuros profissionais o acesso à fala e, assim, conseguir construir técnicas adequadas para lidar com as problemáticas mais difíceis do ser humano ${ }^{12}$. 
Além disso, o jovem médico, desde antes de sua formação médica, carrega sua escala de valores - éticos, estéticos e outros - e não se despe deles na relação profissional com o paciente ${ }^{13}$. Daí decorre que a consciência do aluno de seus valores e de sua natureza humana filtrará qualquer experiência, seja programada no currículo escolar, seja estranha a ele. Portanto, o passo inicial de qualquer programa de formação humanística deve desenvolver no aluno a consciência de seus próprios valores, de sua própria humanidade ${ }^{13}$.

No curso de Medicina da Uece, há inserção de disciplinas voltadas para a discussão de conteúdos que incluem os determinantes sociais, culturais, comportamentais, psicológicos, éticos e legais, nos níveis individual e coletivo do processo saúde-doença. O grupo Humanidades, Saberes e Práticas em Saúde nasce em 2004, como núcleo de desenvolvimento de pesquisas, composto por estudantes de Medicina, sob a orientação de um professor-doutor, que pretende explorar como a prática médica lida com as experiências de pacientes, médicos, saúde, doença e sofrimento, lembrando que a abordagem das humanidades em saúde prevê a incorporação de elementos das ciências humanas.

Este trabalho apresenta as áreas de atuação e as atividades realizadas pelo grupo, abordando a relação médico-paciente por meio de uma visão biopsicossocial.

\section{METODOLOGIA}

Atualmente, o grupo Humanidades tem nove membros: oito acadêmicos do curso de Medicina da Uece (seis do décimo semestre, um do sétimo e um do quinto), liderados por um orientador professor adjunto desta Universidade, com graduação em Medicina e Cirurgia pela Faculdade de Medicina da Universidade de Modena, na Itália, e doutorado em Antropologia pela Universidade de Montreal. O grupo tem como linhas de ação o desenvolvimento de trabalhos científicos na área de relação médico-paciente e o exercício de atividades de ensino do mesmo tema no terceiro e quarto semestres do curso de Medicina. Visa ao crescimento pessoal e intelectual de seus integrantes, contribuindo para a formação de um profissional atuante, com pensamento crítico e espírito científico. Aplica metodologias ativas de aprendizagem, entre as quais trabalho em pequenos grupos, centradas nos participantes, assim como role play, fortalecendo a possibilidade de alcance dos objetivos propostos.

O grupo surgiu há quatro anos como uma necessidade de seus integrantes. Quando as disciplinas clínicas foram introduzidas na vida acadêmica, buscaram-se oportunidades para a prática de atividades extracurriculares, com o intuito de alcançar uma formação global em que fossem aperfeiçoadas as habilidades de comunicação na entrevista clínica.

A contribuição de cada um dos membros e as atividades desenvolvidas no âmbito da Humanização em Saúde trazem ao grupo um estilo dinâmico que se reflete, diretamente, no desenvolvimento de seus integrantes, trazendo acréscimos fundamentais para a sua formação. A inserção de novos membros se dá por procura espontânea de acadêmicos de Medicina que se interessem pelo tema, o que acarreta uma renovação com inter-relações fortes e construtivas. A todos os membros dá-se o direito de obter bolsas de iniciação científica, o que determina uma ligação mais próxima dos acadêmicos com as atividades de pesquisa. Com o intuito de proporcionar ao grupo um caráter mais oficial dentro da Universidade, está em andamento o projeto de torná-lo grupo de extensão.

O orientador tem a missão de estimular a aprendizagem ativa de seus membros por meio de vivências, reflexões e discussões, contando, eventualmente, com a colaboração de outros docentes para desenvolver suas atividades. A eficácia da liderança do orientador depende dos fatores situacionais, natureza da tarefa, organização do grupo, habilidades, personalidade e expectativas. $\mathrm{O}$ orientador exerce papel primordial na condução do grupo, estimulando um processo educacional que estabelece atitude positiva, autoconfiança e desenvolvimento da autonomia dos membros, tornando-os agentes multiplicadores das vivências e dos conhecimentos adquiridos.

Esta colaboração docente-discente na execução de um projeto científico contribui para: formação de uma concepção mais abrangente da realidade científica; reforço do interesse e da inclinação permanente ao conhecimento e ao desenvolvimento de habilidades; aquisição de métodos de trabalho científico; criação de hábitos que contribuem para estreitar as relações entre estudantes e docentes vinculados a um trabalho comum ${ }^{14}$.

O grupo conta com estrutura física própria no campus, onde são realizadas reuniões quinzenais para planejamento de atividades e discussão de temas. Esta unidade dispõe de quatro computadores, todos conectados à internet e com impressora, que constituem uma base importante de dados e de atualização coletiva dos discentes sobre as novidades da literatura, bem como uma forma de facilitar o contato deste grupo com os demais semelhantes em todo o País. Há, ainda, um painel onde os alunos podem expor sua produção bibliográfica na área de interesse do grupo, assim como folhetos sobre eventos científicos, como incentivo à participação nesses encontros para aquisição de 
conhecimentos e divulgação de dados. Dispõe-se de um grupo de $e$-mail pelo qual seus integrantes podem interagir à distância.

\section{RESULTADOS}

As ações do grupo se dividem em atividades de treinamento em sala de aula e pesquisa.

O treinamento, em sala de aula, é realizado pelo grupo por meio do preparo e apresentação de aulas para alunos das disciplinas de Ciências Sociais e Saúde e Iniciação ao Exame Clínico e Relação Médico-Paciente do curso de Medicina da Uece. O grupo participa aproximadamente de 12-24 horas de aulas nas duas disciplinas, que têm 72 horas cada. As dinâmicas de aprendizagem em pequenos grupos e de role play abordaram diferentes aspectos da relação médicopaciente, como: a consulta centrada no paciente, a utilização de perguntas abertas e fechadas, a transmissão de más notícias ${ }^{15}$. Além disso, o grupo organizou um minicurso para os alunos de Medicina que participaram do XXXVI Encontro Científico dos Estudantes de Medicina (Ecem) sobre habilidades de comunicação na relação médico-paciente. Essas atividades contribuem para a troca de experiências e conhecimentos entre os estudantes de Medicina pertencentes à Universidade, no caso das aulas das disciplinas citadas, e de todo o Brasil, no caso do Ecem.

As exposições preparadas pelos integrantes tocam as principais habilidades comunicacionais: acolhimento; capacidades de escuta; formulação de perguntas abertas e fechadas; capacidade de resumir; aconselhamento; como comunicar más notícias. Elas seguem um roteiro, que inclui: 1) introdução acerca do tema; 2) exposição de casos clínicos que provoquem discussão sobre o tema; os casos são discutidos em pequenos grupos de alunos, de seis a oito por grupo; 3) role play; 4) apresentação do modelo teórico e de conceitos novos; 5) conclusão. O grupo possui um banco de casos clínicos baseados em experiências próprias e desenvolvidos segundo um modelo definido ${ }^{16}$. O role play consiste numa dinâmica em que se pede aos alunos que se dividam em trios, em que um representará o médico, outro, o paciente, e o terceiro, o observador. Os alunos, então, encenam a situação proposta e, em seguida, trocam de papéis. Finalmente, discute-se com os alunos o que observaram durante a atividade, utilizando a técnica psicodramática de reativação do conhecimento adquirido.

No que se refere às atividades de pesquisa, os integrantes estão ampliando a amostra de uma pesquisa realizada com médicos oncologistas acerca da visão da relação médico-paciente na consulta oncológica17,18,19. Os dados são obtidos em entrevistas estruturadas que buscam abordar as principais dificuldades do médico frente ao paciente oncológico. O grupo apresentou os resultados encontrados em diversos eventos científicos, sob a forma de banner e trabalho completo, e no momento busca ampliar a pesquisa e elaborar artigo científico para publicação ${ }^{20}$.

A pesquisa, conduzida concomitantemente às atividades de ensino, tem se mostrado bastante produtiva, visto que os achados da primeira reforçam o conteúdo da segunda.

Além dessa pesquisa, realizam-se atividades de revisão bibliográfica e produção de material para publicação. Revisamos artigos sobre os temas: Educação Médica, habilidades comunicacionais, como comunicar más notícias, a relação médico-paciente e humanização.

\section{DISCUSSÃO E CONCLUSÃO}

A criação do grupo de Humanidades, Saberes e Práticas em Saúde se dá num momento propício, refletindo a identidade do curso de Medicina da Uece - um curso novo, cuja missão é formar o médico generalista com sólida fundamentação científica e técnica, dotado de comportamento ético, sentimento de afeição ao semelhante, capacidade analítica e poder criativo na aplicação dos conhecimentos e práticas adquiridos para a tomada de decisões na promoção, manutenção e habilitação da saúde individual e coletiva e na prevenção e tratamento dos transtornos e agravos da saúde.

A criação de um vínculo graduação-pesquisa tem sua importância na medida em que estimula os membros do grupo a manter o senso crítico, produzindo e analisando conhecimentos. Os trabalhos desenvolvidos pelos alunos, por meio da interface pesquisa de campo/aulas para a graduação, se mostraram de grande valia quando comparados com outros trabalhos descritos na literatura, servindo, inclusive, de embasamento teórico para as aulas da graduação. A riqueza dos dados coletados, a partir das experiências de profissionais médicos de instituições conveniadas, serviu não só para enriquecer a experiência pessoal do grupo, como também para reforçar o conteúdo das aulas e minicursos promovidos.

Assim, busca-se aproximar a temática da relação médicopaciente do cotidiano dos estudantes de Medicina, contribuindo para o desenvolvimento de uma atitude humanizada frente ao ser humano portador de enfermidade.

\section{REFERÊNCIAS}

1. Tosteson DC. New Pathways in General Medical Education. N Engl J Med 1990; 322(4): 234-38.

2. Heycox K, Bolzan N. Applying problem-based learning in first-year social work. In: Boud D, Geletti G eds. The challenge of problem based learning. New York: St. Martin's Press; 1991. p. 186-93. 
3. Boelen C. Medical Education Reform: the Need for Global Action. Acad Med 1992; 67(1): 745-49.

4. Nogueira HE. A educação médica e o país real. Médicos. São Paulo: FMUSP; 1998. 30-7.

5. Almeida MJ. A educação médica e as atuais propostas de mudança: alguns antecedentes históricos. Rev Bras Educ Med 2001; 25(2): 42-52.

6. Deslandes S org. Humanização dos cuidados em saúde: conceitos, dilemas e práticas. Rio de Janeiro; Ed. Fiocruz; 2007.

7. Tapajós R. Introducing the arts into medical curricula. Interface 2002; 6(10): 27-36.

8. More AR. Medical humanities: a new medical adventure. N Engl J Med 1976; 295:1479-80.

9. Quintana AM, Cecim PS, Henn CG. O preparo para lidar com a morte na formação do profissional de medicina. Rev Bras Educ Med 2002; 26(3): 204-10.

10. Trindade EMV, Almeida HO, Novaes MRCG, Versiane ER. Resgatando a Dimensão Subjetiva e Biopsicossocial da Prática Médica com Estudantes de Medicina: relato de caso. Rev Bras Educ Med 2005; 29(1): 48-50.

11. Aguiar AC. Implementando as novas diretrizes curriculares para a educação médica: o que nos ensina o caso de Harvard? Interface 2001; 5(8): 161-66.

12. Leite AJM, Caprara A, Caprara JM. Habilidades de comunicação com pacientes e familiares. São Paulo: Sarvier; 2007.

13. Tavares FM. As Contribuições da Medicina Psicossomática à Formação Médica. Rev Bras Educ Med. 2005; 29(1): 64-9.

14. Amador M, Britto JEF, Valido S, Penã M. El trabajo científico de los estudiantes: su papel en la formación de los professionales de la salud. Educ Med Salud 1984; 18(4): 344-57.

15. Fallowfield L, Jenkins, V. Communicating sad, bad, and difficult news in medicine. Lancet 2004; 363(9405): 312-19.

16. Caprara A. A construção narrativa do texto do problema. In: Mamede S, Penaforte J org. Aprendizagem baseada em problemas. São Paulo: Hucitec; 2001. p.143-56.
17. Carvalho AGN, Silva CMGCH, Lino CA, Andrade FC, Gomes IEVM, Augusto KL, et al. O médico e o paciente oncológico: medos, angústias, habilidades comunicacionais. Anais do 35. Encontro Científico de Estudantes de Medicina; 2006 jul. 16-23; Fortaleza, Brasil; 2006

18. Carvalho AGN, Jucá NBH, Lino CA, Silva CMGCH, Andrade FC, Gomes IEVM, et al. Percepção dos médicos frente ao paciente oncológico: dificuldades na relação médico-paciente-família. Congresso Cearense de Cancerologia; 2005 abr. 20-23; Fortaleza, Brasil; 2005.

19. Silva CMGCH, Jucá NBH, Carvalho AGN, Andrade FC, Lino CA, Gomes IEVM, et al. A percepção do médico frente ao paciente oncológico: habilidades comunicacionais na relação médico-paciente.

Anais 57. Reunião Anual da SBPC; 2005 jul. 17-22; Fortaleza, Brasil; 2005.

20. Gomes IEVM, Carvalho AGN, Silva CMGCH, Lino CA, Caprara A. Humanidades, saberes e práticas em saúde: a prática médica e as experiências dos pacientes assistidos pelo programa de saúde da família no município de Fortaleza. 9. Semana Universitária; 2004 out. 18-22; Fortaleza, Brasil: Universidade Estadual do Ceará; 2004.

\section{CONFLITOS DE INTERESSE}

Declarou não haver.

\section{ENDEREÇO PARA CORRESPONDÊNCIA}

Kathiane Lustosa Augusto Universidade Estadual do Ceará - Uece

Grupo Humanidades, Saberes e Práticas em Saúde - CCS Campus do Itaperi

Av. Padre Antônio Tomás, 150 - apto 901

60140-160 - Fortaleza - CE

E-mail: kathianelustosa@yahoo.com.br 